\title{
Eficacia de las técnicas de relajación en la disminución de tensión, ansiedad y estrés percibidos por pacientes con cáncer bajo tratamiento de quimioterapia
}

\author{
Flor Esmeralda Larios-Jiménez ${ }^{1,2}$, Leivy Patricia González-Ramírez ${ }^{3}$, Roberto Montes-Delgado4, \\ Fabiola González-Betanzos ${ }^{5}$, Andrés de Jesús Morán-Mendoza ${ }^{6}$, Pedro Solano-Murillo ${ }^{7}$, \\ Antonio Oceguera-Villanueva ${ }^{8}$, Mónica Montero-Ramírez ${ }^{8}$ y Adrián Daneri-Navarro ${ }^{9 *}$
}

${ }^{1}$ Alumna del Doctorado Interinstitucional en Psicología, Campus Colima, Universidad de Colima, Colima; ${ }^{2}$ Departamento de Disciplinas Filosóficas, Metodológicas e Instrumentales, Centro Universitario de Ciencias de la Salud, Universidad de Guadalajara, Guadalajara; ${ }^{3}$ Centro Universitario de Tonalá, Universidad de Guadalajara, Tonalá; ${ }^{4}$ Facultad de Psicología, Universidad de Colima, Colima; ${ }^{5}$ Facultad de Psicología, Universidad Michoacana de San Nicolás de Hidalgo, Morelia; ${ }^{6}$ UMAE Hospital de Ginecoobstetricia; ${ }^{7}$ UMAE Hospital de Especialidades, CMNO, IMSS; ${ }^{8}$ Instituto Jalisciense de Cancerología; ${ }^{9}$ Departamento de Fisiología, Centro Universitario de Ciencias de la Salud, Universidad de Guadalajara, Guadalajara. México

\begin{abstract}
Resumen
Introducción: El cáncer (CA) es un problema mundial de salud pública. La atención a pacientes con diagnóstico oncológico incluye tratamientos como la quimioterapia, que pueden generar reacciones emocionales negativas. Las técnicas de relajación han mostrado eficacia para disminuir la ansiedad y el estrés en esta población. Material y métodos: Se aplicaron técnicas de respiración diafragmática e imaginación guiada a 552 pacientes en las salas de quimioterapia ambulatoria de tres hospitales de la Zona Metropolitana de Guadalajara. Se evaluó con una escala visual analógica en la cual los pacientes puntuaron sus niveles de tensión, ansiedad o estrés antes y después de la intervención. Resultados: La media de las puntuaciones obtenidas según la escala visual analógica fue de 3.24 (desviación estándar [DE]: 2.8) en la evaluación previa a la técnica de relajación y de 1.2 (DE: 1.9) en la evaluación posterior a la relajación. Se obtuvo una diferencia estadísticamente significativa entre las dos evaluaciones $(p<0.001)$. Se obtuvieron diferencias significativas entre los hospitales $(p<0.001)$. Conclusiones: Nuestros resultados resaltan la eficacia de las técnicas de relajación implementadas en pacientes que reciben quimioterapia, disminuyendo tensión, ansiedad y estrés percibidos. Las técnicas de relajación pueden ser entrenadas y administradas por el personal de salud a los pacientes durante su estancia hospitalaria. La práctica generalizada de estas técnicas por parte de los pacientes mejorará el estado de bienestar al disminuir las afectaciones emocionales causadas por el diagnóstico y los tratamientos.
\end{abstract}

Palabras clave: Respiración diafragmática. Imaginación guiada. Cáncer. Quimioterapia. Programa de navegación de pacientes

\section{Efficacy of relaxation techniques in the reduction of tension, anxiety and stress perceived by patients with cancer under chemotherapy treatment}

\section{Abstract}

Introduction: Cancer constitutes a public health problem worldwide. Attention to patients with cancer includes treatments such as chemotherapy, which may generate emotional negative reactions. Relaxation techniques have shown to reduce 
anxiety and stress in this population. Materials and Methods: 552 patients of the chemotherapy areas of three hospitals of Metropolitan Guadalajara zone were trained in diaphragmatic breathing and guided imagery. Patients were assessed with an visual analogue scale, which scored their tension, anxiety or perceived stress before and after relaxation training. Results: The mean obtained by the visual analogue scale was 3.24 (SD: 2.8) before and 1.2 (SD: 1.9) after relaxation training respectively $(p<0.001)$. Moreover, differences in means among patients in different hospitals $(p<0.001)$ were observed. Conclusions: Our results highlight the effectiveness of relaxation techniques implemented in patients receiving chemotherapy, reducing perceived stress, anxiety and tension. Health professionals can train patients on the use of relaxation techniques during their hospital visits. The generalized practice of these techniques by the patients will improve their state of wellbeing, by reducing the emotional affections caused by cancer diagnosis and the treatments.

Key words: Diaphragmatic respiration. Guided imagination. Cancer. Chemotherapy. Patient navigation program.

\section{Introducción}

El CA es un problema de salud con una elevada mortalidad en los países en vías de desarrollo, debido al diagnóstico tardío ${ }^{1}$. Uno de los tratamientos más utilizados para tratar etapas avanzadas de la enfermedad es la quimioterapia. Ante esta, los pacientes con CA pueden experimentar estrés, ansiedad, miedo, temor, enojo y frustración, entre otras reacciones emocionales; derivadas de una sensación de falta de control de la situación y ante una visón acentuada del CA como una enfermedad sinónimo de muerte ${ }^{2,3}$. Las reacciones emocionales pueden dificultar el proceso de adaptación al tratamiento ${ }^{4,5}$, y sin un tratamiento apropiado y oportuno, pueden culminar en cuadros de depresión, ansiedad, problemas de sueño, baja adherencia terapéutica y afectación de la calidad de vida ${ }^{6,7}$.

El estrés psicológico es el resultado de una relación entre el individuo y un entorno que es evaluado como amenazante o desbordante. Es una apreciación por parte de la persona de que su entorno le exige demasiado, sobrepasa sus recursos y es perjudicial para su bienestar. Hay que tener en cuenta que las diferencias individuales juegan un papel significativo en los procesos de estrés. Características predisponentes, motivaciones, actitudes y experiencias determinan cómo las personas perciben y valoran las demandas. Esta evaluación influye en los estilos de afrontamiento, las respuestas emocionales y, a largo plazo, en los resultados de salud ${ }^{8}$.

En las últimas décadas se han utilizado con gran éxito y como complemento a los tratamientos farmacológicos las técnicas de relajación ${ }^{9,10}$. Particularmente, en pacientes con tratamiento de quimioterapia se han considerado un recurso importante, incluso para síntomas como las náuseas y el vómito ${ }^{11}$. El aprendizaje de estas técnicas es sencillo, sin embargo, se requiere inicialmente del entrenamiento y supervisión por parte del profesional de la salud mediante intervenciones breves. La imaginación guiada y la respiración diafragmática son ampliamente utilizadas para disminuir comorbilidades como el estrés, la ansiedad y la depresión, y por tal motivo fueron seleccionadas para el presente estudio ${ }^{12,13}$. Además, son útiles para: a) disminuir el dolor y las molestias de la tensión muscular, b) reducir la activación simpática, c) lograr un estado de tranquilidad y eliminar la tensión subjetiva, d) conciliar el sueño y el descanso, e) centrarse en las sensaciones corporales del aquí y ahora, y desviar la atención de los pensamientos catastróficos, y g) como estrategia de afrontamiento dar un sentido de control a las situaciones ${ }^{14}$.

La relajación mediante la respiración diafragmática es fácil de aprender y se puede practicar una vez entrenada en cualquier contexto, es una habilidad que requiere prácticas de dos 0 tres veces diarias durante aproximadamente 15020 minutos para conseguir los resultados deseados. Implica llevar a cabo cinco pasos básicos: a) lograr que el aire inspirado se dirija a la parte inferior de los pulmones, b) conseguir que el aire inspirado se dirija a la parte inferior y media de los pulmones, c) alcanzar una inspiración completa, d) obtener una espiración más completa y regular, y e) establecer una adecuada alternancia respiratoria ${ }^{14}$. Por su parte, la imaginación guiada consiste en la creación consciente y voluntaria de representaciones mentales de objetos, lugares, personas, situaciones o de uno mismo.

El objetivo del presente estudio fue evaluar la eficacia de las técnicas de relajación empleadas para disminuir los síntomas de tensión, ansiedad o estrés percibidos en pacientes con CA que recibieron quimioterapia ambulatoria en tres hospitales de Guadalajara Jalisco, México.

\section{MATERIAL Y MÉTODOS}

\section{Participantes y escenarios}

Entre abril de 2014 y noviembre de 2015 se aplicaron las técnicas de respiración diafragmática e imaginación 
guiada a 588 pacientes en las salas de quimioterapia de tres hospitales de la Zona Metropolitana de Guadalajara: Hospital de Especialidades y Hospital de Ginecoobstetricia del Instituto Mexicano del Seguro Social, que atiende a población derechohabiente, así como en el Instituto Jalisciense de Cancerología, cuya población de pacientes atendidos no tiene Seguridad Social. En la sala de quimioterapia del Hospital de Especialidades se ofrecen servicios de administración de medicamentos a pacientes con CA, así como a pacientes con enfermedades reumatoides y del sistema inmunológico; la aplicación de los tratamientos se lleva a cabo en cubículos de máximo tres personas por área. En los otros dos hospitales, las unidades de quimioterapia ofrecen tratamientos solo a población con CA y en estas instituciones las salas son amplias y compartidas por varios pacientes, percibiéndose un ambiente grupal. Para el presente trabajo, 36 evaluaciones fueron excluidas por tratarse de pacientes con Lupus o que no completaron la escala en alguno de los momentos de evaluación.

\section{Procedimiento}

Para la aplicación de las técnicas de relajación fueron entrenados y supervisados nueve aplicadores, de las carreras de psicología, enfermería y medicina, quienes prestaban servicio social o prácticas profesionales dentro del programa de navegación para ayuda de pacientes oncológicos y sus familiares de la Universidad de Guadalajara, denominado en lo sucesivo programa de navegación de pacientes, el cual tiene como objetivo central beneficiar al paciente con CA y a sus familiares facilitando con profesionalidad y calidez la superación de barreras para lograr una atención integral y oportuna, mediante la vinculación interinstitucional y social, servicios como la psicoeducación, el acompañamiento emocional y el entrenamiento en técnicas de relajación, además de contribuir en la formación de recursos humanos en salud promoviendo el trato ético y la mejora de la práctica asistencial mediante la investigación.

Previamente a la aplicación de las técnicas de relajación se establecía un clima de confianza (rapport) con los pacientes que estaban recibiendo su tratamiento con quimioterapia. Enseguida se les explicaban los beneficios de practicar las técnicas de relajación en el proceso oncológico y se les invitaba a participar en la relajación y completar la primera parte de la evaluación para participar en el presente estudio.
Durante la intervención, el facilitador, mediante la técnica de modelamiento, instruía a los participantes para realizar adecuadamente la respiración diafragmática. Una vez que la realizaban correctamente, se les invitaba a cerrar los ojos y a escuchar atentamente la voz del facilitador, que les guiaba por algún escenario en particular, el cual era elegido de acuerdo a las características del grupo o preferencias de las personas con quien se trabajaba. Después, se procedía a narrar las características del escenario elegido (bosque, playa, campo de tulipanes, bosque tropical o nadando con los delfines), modulando la voz, alternando pausas breves y largas de acuerdo a las características del escenario trabajado. Por último, se realizaba la evaluación posterior y se promovía una retroalimentación sobre la experiencia del paciente. Todo el procedimiento tuvo una duración de entre 40 y 60 minutos.

\section{Instrumento}

Se diseñó una escala visual analógica para medir el nivel de tensión, ansiedad o estrés que estaba sintiendo el participante antes y después de la aplicación de la técnica de relajación. Se pensó en un instrumento que fuera sencillo y rápido de contestar. La primera parte solicita información sociodemográfica y clínica como la edad, el lugar de residencia, la escolaridad, el estado civil, el diagnóstico, el ciclo o número de quimioterapia que le están aplicando, y además se indaga sí es la primera vez que se aplica el instrumento o cuántas veces lo ha recibido antes por parte de algún navegador. En las instrucciones de «Antes de la técnica» se invita a puntuar su nivel de tensión, ansiedad o estrés en el momento en una escala de 0 a 10, donde 0 es muy relajado y no siente nada de tensión, ansiedad o estrés, y 10 representa la sensación más grande que haya tenido en su vida de las condiciones señaladas. Posterior a la aplicación de la técnica de relajación, se pide que lean las instrucciones de «después de la técnica» y vuelven a puntuar su nivel de tensión, ansiedad o estrés bajo los mismos criterios. Teniendo la posibilidad de anotar por último de manera narrativa alguna observación o recomendación respecto a la técnica aplicada.

\section{Análisis estadístico}

Los análisis fueron realizados utilizando el programa Statistical Package for Social Sciences (SPSS ${ }^{\circledR}$ ), versión 20.0. Se obtuvieron frecuencias, porcentajes, 
media y DE para describir las características sociodemográficas y clínicas de los pacientes.

Se utilizaron las pruebas de normalidad de Kolmogorov-Smirnov y Shapiro-Wilk, encontrando una distribución normal de los datos. Se realizó una prueba t de Student para datos apareados para comparar las medias de las puntuaciones obtenidas en la evaluación pre y post aplicación de técnica de relajación con la escala visual analógica.

Se creó una variable a partir de las diferencias obtenidas entre las evaluaciones previa y posterior a la aplicación de la relajación. Con ella se realizó una prueba t para muestras independientes para conocer sí existían diferencias en la reducción de tensión, ansiedad o estrés entre los pacientes por sexo y entre aquellos «sin Seguridad Social» y «con Seguridad Social».

\section{Aspectos éticos}

Existe un convenio de colaboración entre la Universidad de Guadalajara y los hospitales participantes para ofrecer diversos servicios a pacientes con CA mediante el programa de navegación de pacientes. De acuerdo con el artículo 11.3 de la NORMA Oficial Mexicana NOM-012-SSA3-2012 ${ }^{15}$, que establece los criterios para la ejecución de proyectos de investigación para la salud en seres humanos en los casos de investigaciones sin riesgo o con riesgo mínimo, el consentimiento informado no es requerido. Se les explicó verbalmente a los pacientes el propósito de publicar los resultados de las evaluaciones, aclarando que la información sería confidencial y explicando que podían retirarse del estudio en cualquier momento. Además, la investigación se dirigió bajo los principios éticos establecidos por la Declaración de Helsinki para la investigación con seres humanos.

\section{RESULTADOS}

La muestra total fue de 552 pacientes con CA. El promedio de edad de los participantes fue de $50 \pm 15$ años. Alrededor del $83 \%$ de la muestra fueron mujeres y el $51 \%$ no contaban con Seguridad Social (Tabla 1). La media de las puntuaciones obtenidas en la escala visual analógica fue de 3.24 (DE: 2.8) en la evaluación previa a la relajación y de 1.2 (DE: 1.9) en la evaluación posterior a la relajación (Fig. 1). Se obtuvo una diferencia estadísticamente significativa entre las medias de estas dos evaluaciones, con una $p<0.001$.

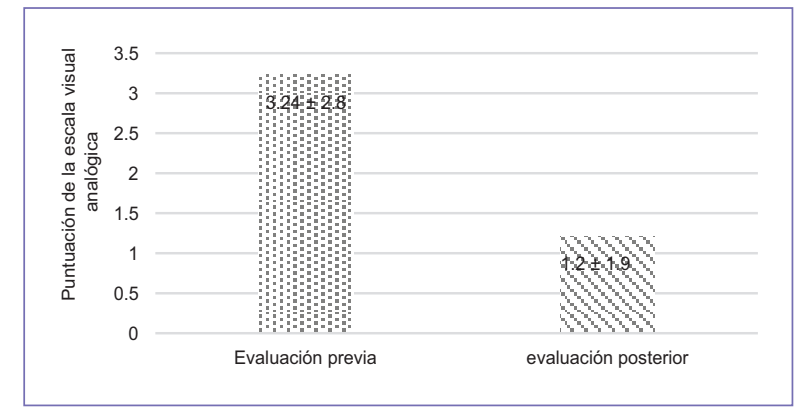

Figura 1. Comparación de puntuaciones antes y después de la utilización de la técnica de relajación.

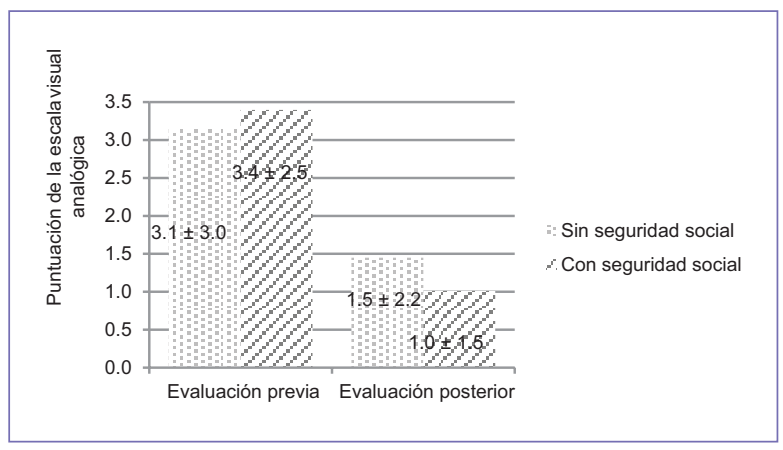

Figura 2. Comparación de los niveles de tensión, ansiedad o estrés percibidos entre personas sin Seguridad Social y con Seguridad Social.

Cabe resaltar que de los 552 pacientes evaluados, 389 (70.5\%) percibieron un nivel menor de tensión, ansiedad y estrés, 149 (27\%) tuvieron la misma puntuación antes y después de la técnica y solamente $14(2.5 \%)$ puntuaron más alto en la percepción de tensión, ansiedad o estrés después de la técnica de relajación.

Con la variable generada con la diferencia entre la evaluación previa y posterior a la técnica de relajación de la escala visual analógica no se obtuvieron diferencias estadísticamente significativas según el sexo $(p=0.11)$. Por el contrario, se encontró significancia estadística entre las pacientes "sin Seguridad Social» $y$ "con Seguridad Social», con un valor de $p<0.001$. Observando por un lado una media de $3.15 \pm 3.04$ en la evaluación previa y de $1.45 \pm 2.17$ en la evaluación posterior de las personas «sin Seguridad Social»; y por otro lado, $3.4 \pm 2.46$ y $1.01 \pm 1.51$ respectivamente en las personas «con Seguridad Social» (Fig. 2).

\section{DISCUSIÓN}

Nuestros resultados muestran una disminución en los niveles de tensión, ansiedad o estrés percibidos en 
Tabla 1. Medidas de tendencia central, dispersión, frecuencias y porcentajes de las características de la muestra

\begin{tabular}{|c|c|c|}
\hline & $\mathrm{m} \pm \mathrm{DE}^{*}$ & n $(\%)^{* *}$ \\
\hline $\begin{array}{l}\text { Edad } \\
\text { Género } \\
\text { Femenino } \\
\text { Masculino } \\
\text { Seguridad Social } \\
\text { Sin Seguridad Social } \\
\text { Con Seguridad Social } \\
\text { Estado civil } \\
\text { Soltero } \\
\text { Casado } \\
\text { Divorciado } \\
\text { Viudo } \\
\text { Unión libre } \\
\text { Ocupación } \\
\text { Empleado } \\
\text { Desempleado }\end{array}$ & $50 \pm 15$ & $\begin{array}{c}122(22.1) \\
348(63) \\
25(4.5) \\
46(8.3) \\
4(0.7)\end{array}$ \\
\hline
\end{tabular}

*media \pm desviación estándar

${ }^{* *}$ Frecuencia (porcentajes)

Los datos perdidos no están especificados en la tabla.

los pacientes oncológicos tras la aplicación de las técnicas de relajación durante la quimioterapia. Dichos pacientes suelen manifestar entre otros síntomas físicos y psicológicos, tensión, ansiedad o estrés durante la aplicación del tratamiento ${ }^{16-18}$ y el estrés en particular se ha asociado con el deterioro de la capacidad del sistema inmunológico para defender al cuerpo ${ }^{19,20}$.

Un estudio experimental que evaluó los beneficios de la práctica diaria, durante una semana, de imaginación guiada en pacientes con CA de mama, mostró que quienes recibían el entrenamiento en relajación manifestaron una disminución significativa en insomnio, doIor, ansiedad y depresión, en contraste con quienes no recibieron dicho entrenamiento, quienes tenían más síntomas de náuseas, vómito y pérdida de apetito, entre otros ${ }^{16}$. Resultados similares se obtuvieron en el estudio de Masafi, Rezaeib y Ahadic ${ }^{21}$, quienes observaron disminución del nivel de ansiedad en el grupo experimental y aumento en el grupo control. Además de realizar entrenamiento en relajación, Masafi, et al. asociaron el uso de biofeedback para intervenir con el grupo experimental de pacientes con CA de mama tras la medición en tres sesiones de quimioterapia.

Por su parte, Charalambous, et al..$^{9}$ realizaron un estudio longitudinal para comprobar la eficacia de la imaginación guiada y la relajación progresiva muscular para el tratamiento de la ansiedad y la depresión en pacientes con CA de mama y de próstata que recibían quimioterapia. Acompañaron sus análisis con el uso de biomarcadores como el cortisol y la enzima amilasa- $\alpha$, encontrando efectiva la combinación de las técnicas para la mediación de la respuesta fisiológica y la disminución de estrés percibido. Estos autores, además, resaltan la evidencia científica sobre alta incidencia de ansiedad y depresión asociadas a los pacientes que reciben quimioterapia, amenazando el cumplimiento del tratamiento y con ello la calidad de vida. Proponen que la intervención de estos pacientes exige un tratamiento más integral, más allá del uso exclusivo de tratamiento farmacológico.

Las técnicas de relajación también han sido probadas como un recurso para el tratamiento del dolor en pacientes oncológicos. Un estudio utilizó la técnica de imaginación guiada para el tratamiento del dolor en pacientes con CA, encontrándola efectiva para dos terceras partes de su muestra. Los pacientes de dicho estudio, además, refirieron el beneficio añadido de la disminución de la ansiedad debida al dolor, puesto que la técnica favorecía la sensación de control sobre este síntoma, contribuía al cambio en la percepción del dolor y a aumentar la eficacia del tratamiento ${ }^{10}$.

Respecto a la respiración diafragmática, Hayama, et al. ${ }^{22}$ realizaron un estudio de evaluación antes/después usando solamente el entrenamiento en la relajación diafragmática, en mujeres diagnosticadas con algún tipo de CA ginecológico que recibían quimioterapia adyuvante. Veinticinco pacientes conformaron su muestra y fueron divididas en dos grupos. Uno de los grupos recibió el entrenamiento de respiración diafragmática. Las puntuaciones en ansiedad-tensión y fatiga en las pacientes del grupo experimental fueron significativamente menores en la evaluación postest que las del grupo control. Por su parte, Qing-Hua Song, et al..$^{23}$ utilizaron la respiración diafragmática acompañada de la relajación progresiva muscular y reportaron un estudio experimental que mostró que el entrenamiento en estas técnicas reduce las reacciones psicológicas adversas como la ansiedad.

Destaca en los estudios antes señalados que son los países de Oriente los que promueven en mayor medida el uso de técnicas de relajación. Nuestro estudio contribuye a sensibilizar en Occidente los beneficios que el entrenamiento en estas técnicas tiene sobre los pacientes con $\mathrm{CA}$ que reciben quimioterapia.

Respecto a las diferencias estadísticamente significativas de los pacientes con Seguridad Social en relación a los que no la tienen, es importante señalar que en el primer escenario algunos pacientes reciben el tratamiento en salas individuales o en un contexto de hasta un máximo de tres personas, lo que propicia un 
clima de mayor privacidad. El aislamiento durante la aplicación de las técnicas de relajación favorece un ambiente de tranquilidad y puede contribuir a disminuir distractores que alteren la concentración. Desafortunadamente, debido a la gran demanda de atención en los hospitales públicos de tercer nivel de nuestro país, en ocasiones la privacidad durante la aplicación del tratamiento no es viable.

El presente estudio presenta algunas limitaciones. En primer lugar, la falta de grupo control, que impide contrastar las diferencias en las puntuaciones de la evaluación pretest y postest. En segundo lugar, no reporta el seguimiento de los pacientes que recibieron las técnicas de respiración diafragmática e imaginación guiada. Actualmente, como parte de un proyecto de evaluación de eficacia de las acciones del programa de navegación, se está trabajando en este punto; por un lado, para conocer si la recomendación de practicar estas técnicas en casa $u$ otros ambientes son seguidas por los pacientes y, por otro lado, para comprobar el efecto a largo plazo del uso de estas técnicas de relajación. Por último, la existencia de múltiples aplicadores pudiera ser una variable confusora, por tanto, consideramos que vale la pena hacer un seguimiento de manera más rigurosa y controlada, para evitar, por ejemplo, que las diferencias entre los hospitales sean debidas también al aplicador de la técnica.

\section{CONCLUSIÓN}

Nuestro estudio se suma a la evidencia científica para promover el entrenamiento al personal de salud sobre la aplicación de estas técnicas, con el objetivo de mejorar el cuidado y la atención integral a los pacientes con $\mathrm{CA}$ que reciben tratamiento con quimioterapia. Además, demuestra la eficacia de las técnicas de relajación para disminuir tensión, ansiedad o estrés percibidos durante la aplicación de quimioterapia. Estudios experimentales ya han comprobado la eficacia de la relajación en el tratamiento del dolor, las náuseas, el vómito, el insomnio y otros síntomas en muestras similares. Las diferencias en la disminución de la tensión, ansiedad o estrés entre los distintos hospitales de nuestro estudio sugieren la influencia del contexto para facilitar la aplicación de las técnicas, siendo preferibles ambientes más privados que disminuyan la probabilidad de distractores o interrupciones durante la práctica.

Las técnicas de relajación son estrategias que pueden ser entrenadas y administradas por el personal de salud interesado, con las ventajas de no ser costosas ni demandantes de tiempo, además de que aportan al bienestar en este y otros contextos de atención hospitalaria, pudiendo incrementar en el usuario la percepción de atención de calidad por parte del equipo de salud.

\section{CONFLICTO DE INTERESES}

Los autores declaran no tener ningún conflicto de interés.

\section{AGRADECIMIENTOS}

A la "Cruzada AVON contra el cáncer de mama» por el financiamiento brindado en atención a las mujeres con CA de mama y sus familiares que son atendidos mediante el programa de navegación de pacientes.

A Susan G. Komen for the CURE $^{\circledR}$ (Grant Number SPMEX1201) por el apoyo financiero para el diseño e implementación del programa de navegación de pacientes en Guadalajara, México.

A la Red Latinoamericana de Investigación en Cáncer y al Instituto Nacional de Cáncer de los Estados Unidos, así como a los Institutos Nacionales de Salud.

Al Consejo Nacional de Ciencia y Tecnología a través del Doctorado Interinstitucional en Psicología de la Universidad Autónoma de Aguascalientes, Universidad de Colima, Universidad de Guadalajara, Universidad de Guanajuato y Universidad Michoacana de San Nicolás de Hidalgo.

Así como a los navegadores Sara Karen Rivas Medina, Lizeth Castellón Guerrero, Carina García Martínez, Alan Tiscareño Rodríguez, Daniel Díaz López, Oscar Daniel Valdez Pulido, Ruth Paulina Aguilar Rivera, Jacqueline Ramírez, Omar Víctor Ernesto Rubio Plascencia y Alma Guillermina Maya González.

\section{BIBLIOGRAFÍA}

1. Torre LA, Bray F, Siegel RL, Ferlay J, Lortet-Tieulent J, Jemal A. Global cancer statistics, 2012. CA Cancer J Clin. 2015;65(2):87-108.

2. Whitaker KL, Cromme S, Winstanley K, Renzi C, Wardle J. Emotional responses to the experience of cancer 'alarm' symptoms. Psychooncology. 2016;25(5):567-73.

3. Ashing-Giwa KT, Padilla G, Tejero J, Kraemer J, Wright K, Coscarelli A, et al. Understanding the breast cancer experience of women: a qualitative study of African American, Asian American, Latina and Caucasian cancer survivors. Psychooncology. 2004;13(6):408-28.

4. Grassi L, Berardi MA, Ruffilli F, Meggiolaro E, Andritsch E, Sirgo A, et al. Role of psychosocial variables on chemotherapy-induced nausea and vomiting and health-related quality of life among cancer patients: a European study. Psychother Psychosom. 2015;84(6):339-47.

5. Meraner V, Gamper EM, Grahmann A, Giesinger JM, Wiesbauer $P$, Sztankay M, et al. Monitoring physical and psychosocial symptom trajectories in ovarian cancer patients receiving chemotherapy. BMC Cancer. 2012;12:77.

6. Ates O, Soylu C, Babacan T, Sarici F, Kertmen N, Allen D, et al. Assessment of psychosocial factors and distress in women having adjuvant endocrine therapy for breast cancer: the relationship among emotional distress and patient and treatment-related factors. Springerplus. 2016;5:486. 
7. Miles A, McClements PL, Steele RJ, Redeker C, Sevdalis N, Wardle J. The psychological impact of a colorectal cancer diagnosis following a negative fecal occult blood test result. Cancer Epidemiol Biomarkers Prev. 2015;24(7):1032-8.

8. Lazarus SR, Kanner AD, Folkman S. Emotions: A cognitive-phenomenological analysis En: Plutchik R, Kellerman H, editores. Theories of Emotion. EE.UU.: Elsevier; 1980.

9. Charalambous A, Giannakopoulou M, Bozas E, Paikousis L. A randomized controlled trial for the effectiveness of progressive muscle relaxation and guided imagery as anxiety reducing interventions in breast and prostate cancer patients undergoing chemotherapy. Evid Based Complement Alternat Med. 2015;2015:270876.

10. Kwekkeboom KL, Hau H, Wanta B, Bumpus M. Patients' perceptions of the effectiveness of guided imagery and progressive muscle relaxation interventions used for cancer pain. Complement Ther Clin Pract. 2008;14(3):185-94

11. Haro LM, Mondéjar R, Muñoz MM, Molina MJ, Olaverri A, Santiago JA. Tratamiento psicológico de las náuseas y vómitos anticipatorios inducidos por quimioterapia o radioterapia. Psicooncología. 2013;10(2-3):289-98.

12. Sleight AG, Duker LI. Toward a broader role for occupational therapy in supportive oncology care. Am J Occup Ther. 2016;70(4):7004360030p1-8.

13. Jensen W, Bialy L, Ketels G, Baumann FT, Bokemeyer C, Oechsle K Physical exercise and therapy in terminally ill cancer patients: a retrospective feasibility analysis. Support Care Cancer. 2014;22(5):1261-8.

14. Cruzado JA. Tratamiento psicológico en pacientes con cáncer. España: Síntesis; 2010.

15. NORMA Oficial Mexicana NOM-012-SSA3-2012, Que establece los criterios para la ejecución de proyectos de investigación para la salud en seres humanos. Secretaría de Salud, 2012
16. Chen SF, Wang HH, Yang HY, Chung UL. Effect of relaxation with guided imagery on the physical and psychological symptoms of breast cancer patients undergoing chemotherapy. Iran Red Crescent Med J. 2015;17(11):e31277.

17. Polat U, Arpaci A, Demir S, Erdal S, Yalcin S. Evaluation of quality of life and anxiety and depression levels in patients receiving chemotherapy for colorectal cancer: impact of patient education before treatment initiation. J Gastrointest Oncol. 2014;5(4):270-5.

18. Pandey M, Sarita GP, Devi N, Thomas BC, Hussain BM, Krishnan R. Distress, anxiety, and depression in cancer patients undergoing chemotherapy. World J Surg Oncol. 2006;4:68.

19. De la Roca-Chiapas JM, Barbosa-Sabanero G, Martinez-Garcia JA, Martinez-Soto J, Ramos-Frausto VM, Gonzalez-Ramirez LP, et al. Impact of stress and levels of corticosterone on the development of breast cancer in rats. Psychol Res Behav Manag. 2016;9:1-6.

20. Segerstrom SC, Miller GE. Psychological stress and the human immune system: a meta-analytic study of 30 years of inquiry. Psychol Bull. 2004;130(4):601-30.

21. Masafia S, Rezaeib O, Ahadic H. Efficacy of biofeedback associated with relaxation in decreasing anxiety in women with breast cancer during chemotherapy. Procedia - Social and Behavioral Sciences. 2011; 30:143-8.

22. Hayama Y, Inoue T. The effects of deep breathing on 'tension-anxiety' and fatigue in cancer patients undergoing adjuvant chemotherapy. Complement Ther Clin Pract. 2012;18(2):94-8.

23. Song QH, Xu RM, Zhang QH, Ma M, Zhao XP. Relaxation training during chemotherapy for breast cancer improves mental health and lessens adverse events. Int J Clin Exp Med. 2013;6(10):979-84. 